\title{
RANGO DE SUFICIENCIA NUTRIMENTAL FOLIAR PARA EL GUAYABO (Psidium guajava L.), EN LA REGIÓN ORIENTE DE MICHOACÁN, MÉXICO
}

\author{
LEAF NUTRIENT SUFFICIENCY RANGE FOR GUAVA (Psidium guajava \\ L.), IN THE EASTERN REGION OF MICHOACAN, MEXICO
}

\section{Víctor H. Volke-Haller ${ }^{1 *}$, Prometeo Sánchez-García ${ }^{1}$, José I. Cortés-Flores ${ }^{1}$, Mauricio Orozco-Méndez ${ }^{2}$ y Ramón Camacho-Blas ${ }^{2}$}

\begin{abstract}
'Postgrado en Edafología, Campus Montecillo, Colegio de Postgraduados. km 36.5 carretera México-Texcoco. 56230, Montecillo, Estado de México, México. ${ }^{2}$ Facultad de Ciencias Agrícolas V-IV, Universidad Autónoma de Chiapas. 30660, Huehuetán, Chiapas, México.

*Autor para correspondencia ( vvolke@colpos.mx.)
\end{abstract}

\section{RESUMEN}

El guayabo (Psidium guajava L.) es un cultivo importante en la región oriente de Michoacán, México, donde cubre una superficie de 9260 ha, y uno de los problemas que presenta es la deficiente nutrición. En relación con el estado nutrimental del guayabo, se determinó el rango de suficiencia nutrimental, así como el efecto de factores del suelo y de manejo del cultivo que inciden en la concentración de nutrientes en el árbol en la región oriente de Michoacán, México. En 31 huertos en 2006, 37 en 2008 y 39 en 2011 se tomaron muestras de suelo para caracterización física y química, y de hoja de los árboles durante la etapa de 25 a $50 \%$ de floración para determinar las concentraciones de nutrientes, y se recopiló información de manejo del cultivo y rendimiento de fruto del árbol. Se ajustaron modelos de regresión para estimar el rendimiento como función de las concentraciones foliares de nutrientes y modelos de éstas como función de las cantidades de nutrientes aplicadas como fertilizante, contenidos de nutrientes y propiedades físicas y químicas del suelo, y factores de manejo del cultivo. Las concentraciones de nutrientes en la hoja presentaron relación con: 1) la respectiva fertilización para $\mathrm{N}, \mathrm{P}, \mathrm{K}, \mathrm{Ca}, \mathrm{Mn}$ y Cu; 2) los contenidos de nutrientes del suelo, para todos los nutrientes evaluados, excepto $B ; y, 3$ ) algunos factores de suelo y de manejo del cultivo que favorecieron o desfavorecieron la absorción de nutrientes por la planta. El rango de suficiencia nutrimental foliar adecuado se asoció con contenidos de nutrientes moderadamente altos y altos del suelo, y las cantidades de fertilizante recomendadas a aplicar en los huertos de la región, para N, P, K, Ca, Mn y Cu.

Palabras clave: Psidium guajava, concentración de nutrientes en la hoja, contenido de nutrientes del suelo, factores de manejo del huerto.

\section{SUMMARY}

Guava (Psidium guajava L.) is an important crop in the Eastern region of Michoacán, Mexico, where it covers 9260 ha, and one of the problems presented is poor nutrition. In relation to the nutritional status of guava, the nutrient sufficiency range was determined, as well as the effect of soil and orchard management factors affecting nutrient nutrient status of trees in the Eastern region of Michoacán, México. Soil samples were taken for physical and chemical characterization, and leaf samples at 25 to $50 \%$ flowering stage to determine nutrient concentrations 31 orchards in 2006, 37 in 2008 and 39 in 2011. In addition, information on orchard management and fruit yield was collected. Regression models were adjusted to estimate yield as a function of nutrient foliar concentration, and for these concentrations as a function of nutrient amounts applied as fertilizer, nutrient content and soil chemical properties, and orchard management factors. The nutrient concentrations in the leaf were related to: 1) the respective fertilization for $\mathrm{N}, \mathrm{P}, \mathrm{K}, \mathrm{Ca}, \mathrm{Mn}$ and $\mathrm{Cu}$; 2) nutrient content of the soil for all nutrients, except for $\mathrm{B}$, and 3) some soil and orchard management factors that increased or decreased the nutrient uptake by the plant. The adequate foliar nutrient sufficiency range was associated with moderately high and high soil nutrient contents, and fertilizer amounts recommended to be applied in orchards for $\mathrm{N}, \mathrm{P}, \mathrm{K}, \mathrm{Ca}, \mathrm{Mn}$ and $\mathrm{Cu}$.

Index words: Psidium guajava, leaf nutrient concentration, soil nutrient content, orchard management factors.

\section{INTRODUCCIÓN}

En 2014 la superficie plantada con guayabo (Psidium guajava L.) en México fue de 20,899 ha, de las cuales 9260 se ubicaban principalmente en la región oriente de Michoacán, en los municipios de Jungapeo (2700 ha), Juárez (2090 ha), Zitácuaro (1560 ha), Susupuato (610 ha), Tuxpan (219 ha), Tuzantla (180 ha) e Hidalgo (24 ha) (SIAP, 2014).

Los rendimientos medios de fruto obtenidos en 2014 fueron de $16.3 \mathrm{t} \mathrm{ha}^{-1}$ en Juganpeo, $16.6 \mathrm{t} \mathrm{ha}^{-1}$ en Juárez, $18.3 \mathrm{t} \mathrm{ha}^{-1}$ en Zitácuaro, $16.2 \mathrm{t} \mathrm{ha}^{-1}$ en Susupuato y 13.3 t ha-1 en Tuzantla (SIAP, 2014), que pueden considerarse relativamente bajos; tal es así, que información de campo obtenida de 107 huertos en los ciclos de producción de 2006, 2008 y 2011 indica variaciones de los rendimientos de 7 a $36 \mathrm{t} \mathrm{ha}^{-1}$, con $31 \%$ de productores que obtuvieron rendimientos menores de 20 t ha $^{-1}$ en árboles de 10 o más años de edad (Volke-Haller et al., 2012).

Entre las causas del bajo rendimiento de huertos de guayabo se encuentran una escasa floración y cuajado de fruto, las deficiencias en el control de maleza, plagas y enfermedades, y el manejo inadecuado de la fertilización, además de algunas condiciones de suelos someros y escasa disponibilidad de agua para riego (Volke-Haller et al., 2012). 
Para el cálculo de la cantidad de fertilizantes a aplicar a los cultivos se suele utilizar el análisis de suelo; no obstante, en árboles frutales éste no siempre es el mejor indicador de la disponibilidad de los nutrientes, pues la absorción por el árbol también depende de otras propiedades químicas, físicas y biológicas del suelo, del volumen de suelo explorado por las raíces, de elementos del clima como la humedad y temperatura, y del manejo del cultivo (Bates, 1971; Jones, 1985; Mills y Jones, 1996; Singh y Singh, 2007).

En árboles frutales, si bien un programa de fertilización no excluye el análisis de suelo, es el análisis de tejido vegetal, usualmente las hojas, el que se utiliza para diagnosticar el estado nutrimental del árbol, y determinar qué elementos pueden encontrarse deficientes. Para un nivel deficiente, cuando el muestreo de hojas se realiza en las primeras etapas de crecimiento del árbol, se podrán hacer las correcciones en el mismo ciclo de producción mediante fertilización; mientras que, cuando se realiza en etapas avanzadas de crecimiento, las correcciones podrán hacerse en el siguiente ciclo de producción (Kotur et al., 1997; Mills y Jones, 1996; Reuter et al., 1997; Singh y Singh, 2007).

Para el uso del análisis foliar se requiere determinar previamente la concentración nutrimental adecuada, con la que el árbol alcanza el rendimiento y calidad de frutos óptimos. Si bien se ha establecido la concentración nutrimental adecuada en árboles frutales para los distintos nutrientes en diversas regiones, que pueden ser extrapoladas a otras, su uso es de tipo general, ya que en los frutales, aunque la concentración nutrimental depende de las características genéticas de la especie, ésta es influenciada por el portainjerto, la variedad, las condiciones del suelo, clima y manejo del árbol (Bates, 1971; Mills y Jones, 1996; Singh y Singh, 2007; Trajano Maia et al., 2007), de tal manera que las concentraciones nutrimentales adecuadas para alcanzar los rendimientos óptimos pueden variar entre regiones (Anjaneyulu y Raghupathi, 2009; Hundal et al., 2007; Volke-Haller et al., 2012).

Lo anterior indica que las concentraciones nutrimentales deban ser relacionadas (calibradas) con el rendimiento para cada variedad y portainjerto, y las condiciones de producción de suelo, clima y manejo. Para ello existen varios métodos; uno es el denominado rango de suficiencia, que con base en la relación entre el rendimiento y la concentración nutrimental en la hoja, considera los siguientes rangos: deficiente, bajo o marginal, adecuado o suficiente, alto y excesivo o tóxico. El rango adecuado o suficiente indica los valores con los cuales se obtienen los máximos rendimientos y óptima calidad del fruto (Mills y Jones, 1996; Sánchez et al., 2012). Una vez establecido el rango adecuado o suficiente se determina, en caso de existir alguna deficiencia nutrimental, la cantidad de fertilizante que opti- mice el rendimiento y calidad del fruto (Mills y Jones, 1996; Sánchez et al., 2012).

En México existe escasa información acerca de la relación entre el rendimiento y la concentración foliar de nutrientes en guayabo, al igual que para la corrección de posibles deficiencias mediante fertilización a partir del análisis foliar. Al respecto, Otero et al. (1997), en guayabo variedad Media China en condiciones de invernadero, encontraron un incremento de $\mathrm{N}$ en las hojas debido a aplicaciones foliares periódicas y al suelo de $\mathrm{KNO}_{3}$ y $\mathrm{NH}_{4} \mathrm{NO}_{3}$ desde antes de la brotación de las hojas hasta la etapa de formación del fruto, que alcanzan valores máximos de $16.6 \mathrm{~g} \mathrm{~kg}^{-1} \mathrm{con}$ el $\mathrm{KNO}_{3}$ y de 21.1 y $18.4 \mathrm{~g} \mathrm{~kg}^{-1}$ con el $\mathrm{NH}_{4} \mathrm{NO}_{3}$ aplicado de manera foliar y al suelo, respectivamente, en la etapa de diferenciación floral. Castelán-Estrada y Becerril-Román (2004), en guayabo variedad Media China en condiciones de invernadero, encontraron un incremento de las concentraciones de $\mathrm{Cu}$ y $\mathrm{Mn}$ en las hojas debido a aplicaciones foliares periódicas de microelementos en el periodo desde la brotación de las hojas hasta la aparición de los botones florales, hasta valores de 116 a $119 \mathrm{mg} \mathrm{kg}^{-1}$ y de 115 a 158 $\mathrm{mg} \mathrm{kg}^{-1}$, respectivamente, a la vez que dichas aplicaciones incrementaron la concentración de Fe, a valores de 111 a $128 \mathrm{mg} \mathrm{kg}^{-1}$; por su parte, las aplicaciones de nitrato de amonio no incrementaron significativamente la concentración de N, con valores de 13.6 a $14.0 \mathrm{~g} \mathrm{~kg}^{-1}$.

Damián-Nava et al. (2006) estudiaron la dinámica y el diagnóstico nutrimental del guayabo variedad Media China, de cuatro años de edad en condiciones de temporal y riego de auxilio, en Iguala, Guerrero. La dinámica nutrimental indicó variaciones en las concentraciones nutrimentales en la hoja durante el ciclo de crecimiento. Para el diagnóstico nutrimental probaron los enfoques de Nivel Crítico, Desviación del Óptimo Porcentual y Sistema Integrado de Diagnóstico y Recomendación (DRIS, por sus siglas en inglés), encontraron que el DRIS presentó la mayor correlación ( $r=$ 0.966, $P=0.01$ ) entre el rendimiento y el índice de desbalance nutrimental, de modo que resultaría apropiado para diagnosticar el estado nutrimental en guayabo con fines de establecer recomendaciones de fertilización.

Adicionalmente, estos autores mencionan las normas DRIS obtenidas con 196 observaciones de análisis foliar y rendimiento, en huertos de guayabo de Calvillo, Aguascalientes y Apozol, Zacatecas. Volke-Haller et al. (2012), en 107 huertos de guayabo variedad Media China de edades entre 4 y 26 años en condiciones de riego en la región oriente de Michoacán, determinaron las concentraciones de N, P, K, Ca, Mg, Fe, Mn, Cu, Zn y B en hojas recientemente maduras en el estado de 25 a 50 \% de floración, en términos de bajas, suficientes y altas según los estándares proporcionados por Marchal (1984) y Mills y Jones (1996), 
con lo que estimaron el estado nutrimental de los árboles y generaron una primera aproximación de recomendaciones de fertilización para su aplicación al suelo o al follaje en dicho estado fenológico.

El objetivo de la presente investigación fue establecer el rango de suficiencia nutrimental de los distintos nutrientes para el guayabo, e identificar los factores de suelo y de manejo del cultivo que inciden en el estado nutrimental del árbol, en la región oriente del estado de Michoacán.

\section{MATERIALES Y MÉTODOS}

\section{Área de estudio}

El estudio se realizó en los ciclos de producción de 2006, 2008 y 2011 en la región oriente del estado de Michoacán, que incluye los municipios de Jungapeo, Juárez y Zitácuaro, con coordenadas de $19^{\circ} 12^{\prime}$ a $19^{\circ} 29^{\prime}$ latitud $N$ y de $100^{\circ} 23^{\prime}$ a $100^{\circ} 30^{\prime}$ longitud O. En la zona productora de guayaba, la altitud varía entre 1050 y 1800 m en Jungapeo, entre 1150 y 1700 m en Juárez, y entre 1500 y 2150 m en Zitácuaro (Mendoza et al., 2005).

En la región se observan los climas: $(A) C\left(W_{1}\right)(w)$, de tipo semicálido subhúmedo con lluvias en verano en Jungapeo, Juárez y una porción de Zitácuaro; Awo(w), de tipo subhúmedo con lluvias en verano, en Juárez y una parte de Jungapeo, y $C\left(w_{1}\right)(w)$ y $C\left(w_{2}\right)(w)$, de tipo templado húmedo con lluvias en verano, en Zitácuaro (Medina et al., 1998; Sánchez, 2007).

En la mayor parte de la región, para altitudes de 1300 a 1909 msnm las precipitaciones medias anuales varían entre 770.9 y 989.9 mm con 75.4 a $83.6 \%$ concentradas de junio a septiembre, y en el área de Tuzantla, con una altitud de $660 \mathrm{~m}$, las precipitaciones medias son de $1274.3 \mathrm{~mm}$, con $88.2 \%$ de junio a septiembre (SMN, 2014).

Según el Sistema de Clasificación FAO-UNESCO, los suelos predominantes en la región son: Leptosoles, con presencia de piedras en el perfil y en superficies accidentadas, en Jungapeo, Juárez y Zitácuaro; Andosoles, en Juárez y Zitácuaro; Luvisoles, en Juárez, Jungapeo y Zitácuaro, y Vertisoles, en Juárez y Jungapeo (INEGI, 2009).

La información recabada del cultivo del guayabo en la región es la siguiente: 1) la poda se hace de enero a abril $(70.5 \%)$, mayo a agosto (16.1\%) y septiembre a diciembre $(14.2 \%)$; 2) el ciclo de producción varía en función de la altitud de 195 a 322 d, lo que condiciona que sea necesario regar los huertos en el periodo sin o con escasas precipitaciones; 3 ) el intervalo entre riegos más frecuente varía de 10 a $15 \mathrm{~d}$, con valores que pueden ser de hasta $20 \mathrm{~d}$ en la época de lluvias, y la lámina de riego varía según el tiempo de riego, con valores de hasta $5 \mathrm{~cm}$; 4) durante el ciclo de cultivo el control de malezas se realiza de tres a cuatro veces y de 10 a 12 veces el control de plagas-enfermedades, según la duración del ciclo; 5) usualmente se realizan dos fertilizaciones, la primera después de la poda y antes del primer riego y la segunda en las primeras etapas de crecimiento del fruto; 6$)$ las fuentes de fertilizantes más frecuentes son: para N, sulfato de amonio ( $21 \%$ de N); para P y Ca, superfosfato de calcio simple ( $20 \%$ de $\mathrm{P}_{2} \mathrm{O}_{5}, 18$ a $21 \%$ de $\mathrm{Ca}$ ); para $\mathrm{K}$, cloruro de $\mathrm{K}\left(60 \%\right.$ de $\left.\mathrm{K}_{2} \mathrm{O}\right)$; y microelementos como Fermil granulado (Mg, 8.0 \%; Mn, 1.2 \%; Zn, $3.1 \%$; $\mathrm{Cu}, 0.1$ \%; Fe, $2.1 \%$; Ca, $3.8 \%$, B, $0.4 \%$; S, $9.2 \%$ ) (Mendoza et al., 2005; Sánchez, 2007; Volke-Haller et al., 2012).

\section{Obtención de la información}

La información se obtuvo en huertos podados de enero a mayo, que es el periodo de poda para la mayoría de los huertos, lo cual implica que se trata de huertos que reciben riego, antes y después del periodo de lluvias.

Se muestrearon al azar 31 huertos comerciales en 2006, 37 en 2008 y 39 en 2011, en los municipios de Jungapeo, Zitácuaro y Juárez, respectivamente. En cada huerto se tomó información de sitio como altitud, pendiente, pedregosidad superficial, color y profundidad del suelo.

Para el muestreo de suelo y hoja, en cada huerto en una superficie aproximada de 1.0 ha se muestrearon seis árboles con base en un muestreo sistemático, según la forma del huerto (Etchevers y Padilla, 2012). La muestra de suelo se obtuvo a una profundidad de 0 a $30 \mathrm{~cm}$, por ser la zona de mayor distribución de raíces absorbentes del árbol en condiciones de riego (Volke-Haller et al., 2012), en tres puntos alrededor del árbol entre el tronco y el bordo del cajete, para formar una muestra compuesta de 16 submuestras. La muestra de hoja se obtuvo en una etapa temprana de crecimiento del árbol, cuando presentaban de 25 a 50 $\%$ de floración, para hacer posible, dado el caso, correcciones de deficiencias, y se colectaron hojas recientemente maduras correspondientes a los pares tercero y cuarto a partir del ápice de la rama, a una altura media de la copa, en cuatro puntos alrededor del árbol, para un total de 16 hojas por árbol y 96 hojas por huerto.

En el suelo se determinó textura, mediante el método de Boyoucos y las siguientes propiedades: a) pH, en relación suelo:agua de 1:2; b) conductividad eléctrica, en extracto de saturación; c) materia orgánica, por combustión húmeda; d) capacidad de intercambio de cationes (CIC) y cationes intercambiables, con acetato de amonio $1 \mathrm{~N}$ a pH de 7, la CIC por destilación y titulación de $\mathrm{H}_{2} \mathrm{SO}_{4}$, el Ca y Mg por titulación con EDTA y el K y Na por espectrofotometría 
de emisión de llama; e) P, por el método de Bray $1 ;$ y, f) microelementos (Fe, Cu, Mn y Zn), extraídos con DTPA y cuantificación por absorción atómica (SEMARNAT, 2002). La interpretación de los resultados se realizó de acuerdo con lo propuesto por Castellanos et al. (2000) para los mismos métodos.

En la hoja se determinó la concentración de $\mathrm{N}$ por microKjeldahl, y de $\mathrm{P}, \mathrm{Ca}, \mathrm{Mg}, \mathrm{K}$, Fe, Mn, Cu, Zn y B, por digestión húmeda con mezcla ácida $\left(\mathrm{HNO}_{3}-\mathrm{HClO}_{4}-\mathrm{H}_{2} \mathrm{SO}_{4}\right)$ y su cuantificación mediante un espectrofotómetro de emisión atómica de inducción acoplada con plasma (Modelo Liberty Serie II Secuencial, Marca Varian®, Alemania) (Alcántar y Sandoval, 1999).

La información acerca del manejo de cultivo se obtuvo por medio de entrevistas directas con los productores, y comprendió: variedad, edad de los árboles, densidad de población, fecha de poda, fecha de floración, riego (fuente de agua, intervalo, número de riegos, duración), fertilización (fuente, número de aplicaciones, oportunidad, dosis de N, P, K, Ca, S y microelementos), control de maleza (método, tipo de herbicida, número de aplicaciones) y de plagas y enfermedades (incidencia, producto, dosis, número de aplicaciones).

El rendimiento de fruto se obtuvo de la información proporcionada por los productores, y se expresó en kilogramos por árbol debido a que los huertos varían en su densidad de población, presentándose con una mayor frecuencia entre 330 y 400 árboles/ha.

El análisis de la información se hizo para cada nutriente de manera separada, y comprendió los siguientes pasos: 1) se ajustó un modelo de regresión del rendimiento como función de la concentración nutrimental en la hoja y de factores de suelo y manejo del cultivo que no tuviesen relación con la nutrición del árbol, para determinar la relación entre el rendimiento y la concentración nutrimental en la hoja; 2) con estos modelos de regresión se calculó el rendimiento alcanzado con la concentración nutrimental en la hoja, en el intervalo de valores observados, a fin de determinar el rango de la concentración nutrimental como porcentaje del rendimiento máximo (deficiente: < $90 \%$, bajo: 90 a 95 $\%$, adecuado: 95 a $100 \%$, alto: 95 a 90 \%, excesivo: < $90 \%$ ); 3) se ajustó un modelo de regresión de la concentración nutrimental en la hoja como función de los factores que pudiesen afectar la absorción del nutrimento por el árbol; esto es, el contenido nutrimental del suelo, las propiedades físicas y químicas del suelo, los factores de manejo del cultivo y las cantidades de nutrientes aplicados en la primera fertilización de N, P, K, Ca y S (después de la poda y antes del primer riego), puesto que son los nutrientes que pudiesen estar relacionados con las concentraciones nutrimentales; 4) con estos modelos de regresión para los nutrientes que se aplicaron como fertilizantes en la primera fertilización, se calculó la cantidad de nutriente con la cual se obtenía la concentración nutrimental adecuada en la hoja, de tal manera que ésta fuera la fertilización a aplicar para obtener el rendimiento óptimo; 5) para los nutrientes que no fueron aplicados en la primera fertilización, sólo fue posible observar si el contenido del nutriente en el suelo o algún otro factor de suelo y de manejo del cultivo tenía efecto sobre la concentración nutrimental en la hoja. Para la obtención de los modelos de regresión se siguió la metodología propuesta por Volke-Haller (2008) para información obtenida mediante muestreo.

\section{RESULTADOS Y DISCUSIÓN}

\section{Factores de sitio, propiedades y contenidos nutrimentales del suelo y factores de manejo del cultivo}

Los huertos presentaron los valores de los factores de sitio que se describen en el Cuadro 1. Por otro lado, la mayor parte de los huertos presentaron suelos arcillosos, con contenidos de arcilla entre 31 y $70 \%$ (87.1\%), contenidos de materia orgánica entre 1 y $4 \%$ (95.4\%), pH de 7.0 o menor (85.3\%) y conductividad eléctrica menor de 0.40 dS $\mathrm{m}^{-1}(90.2 \%)$ (Cuadro 2).

El contenido de los nutrientes en el suelo presentó diferencias entre huertos, con contenidos bajos, moderadamente bajos, adecuados, moderadamente altos o altos,

Cuadro 1. Clases de los factores de sitio, y su distribución porcentual en los huertos de guayabo var. Media China en el oriente de Michoacán.

\begin{tabular}{lcccc}
\hline Factor & \multicolumn{4}{c}{ Clase (porcentaje de huertos) } \\
\hline Altitud (msnm) & 1151 a 1250(31.8) & 1251 a $1500(35.6)$ & 1501 a $1750(26.3)$ & 1751 a $2000(6.3)$ \\
Pendiente (\%) & $<6(38.3)$ & 6 a $15(39.3)$ & 16 a $50(19.6)$ & $>51(2.8)$ \\
Pedregosidad superficial(\%) ${ }^{+}$ & Sin (32.7) & Escasa (37.4) & Media (20.6) & Abundante (9.3) \\
Profundidad media (m) & $0.50(6.6)$ & $0.75(29.9)$ & $1.00(28.0)$ & $1.25(35.5)$ \\
Color & 7.5 YR 3/2, pardo oscuro (50.5) & 10 YR 2/1, negro (49.5) & \\
\hline
\end{tabular}

${ }^{\dagger}$ Escasa: alrededor de $10 \%$, media: alrededor de $20 \%$, abundante: alrededor de $35 \%$. 
según el nutriente (Castellanos et al., 2000), en los que resaltaron valores bajos para P $(49.5 \%)$ y K $(40.3 \%)$ y moderadamente altos y altos para $\mathrm{Mg}(88.1 \%), \mathrm{Fe}(91.6 \%), \mathrm{Mn}$ (94.4\%), Cu (92.6\%) y Zn (81.8\%); para Ca predominan los contenidos adecuados (53.1\%) y moderadamente altos (22.9 \%) (Cuadro 3).

Los valores mínimo, máximo y medio del nivel de los factores de manejo de los huertos muestran una variación importante en la región, la cual puede explicar en parte la variación en los rendimientos observados (Cuadro 4).

\section{Concentración nutrimental en la hoja}

La concentración nutrimental en la hoja a $25-50 \%$ de floración de los huertos muestra una variación considerable entre el valor mínimo y máximo (Cuadro 5), lo que puede ser atribuible a diferencias de las condiciones de suelo y clima, manejo de la humedad (riego), control de maleza, y fertilización (Bates, 1971; Malavolta et al., 1997; Mills y Jones, 1996; Singh y Singh, 2007; Trajano Maia et al., 2007), sin considerar la variedad, que es uniforme en la región.

\section{Rendimiento de fruto y concentración nutrimental en la hoja}

Los modelos de regresión del rendimiento en función de la concentración nutrimental en la hoja incluyeron factores de manejo, tales como edad de los árboles, profundidad del suelo, número de riegos, número de aplicaciones para el control de enfermedades y plagas y número de veces que se practicó control de maleza. En el Cuadro 6 se presentan los modelos que consideran la concentración nutrimental en la hoja, sin incluir las variables de los demás factores señalados. En estos modelos el contenido del nutriente del suelo fue excluido por la concentración del nutriente en la hoja, lo que puede interpretarse en el sentido de que, por una parte, la concentración nutrimental en la hoja fue un mejor estimador del estado nutrimental del árbol en relación al rendimiento de fruto, y, por otra, que el contenido nutrimental del suelo puede no ser un estimador adecuado de dicho estado nutrimental. Estos resultados concuerdan con lo observado por diversos autores (Bates, 1971; Jones, 1985; Mills y Jones, 1996; Singh y Singh, 2007).

Con los modelos de regresión se estimó el rendimiento para valores de la concentración nutrimental en la hoja dentro del intervalo de valores observados, a valores óptimos

Cuadro 2. Clases de propiedades del suelo y su distribución porcentual en los huertos de guayabo var. Media China en el oriente de Michoacán.

\begin{tabular}{lccccc}
\hline Propiedad & \multicolumn{5}{c}{ Clase (porcentaje de huertos) } \\
\hline Arena (\%) & $<11(10.2)$ & 11 a $20(24.9)$ & 21 a $30(39.3)$ & 31 a $40(18.3)$ & 41 a $60(7.3)$ \\
Limo (\%) & 11 a $20(11.9)$ & 21 a $30(45.5)$ & 31 a $40(33.3)$ & 41 a $50(9.3)$ & \\
Arcilla (\%) & 21 a $30(12.9)$ & 31 a $40(19.3)$ & 41 a $50(30.7)$ & 51 a $60(27.1)$ & 61 a $70(10.0)$ \\
Materia orgánica (\%) & $<1(4.6)$ & 1 a 2 (33.2) & 2 a 3 (40.3) & 3 a $4(16.4)$ & 4 a $6(5.5)$ \\
pH & 5.1 a 5.5(13.8) & 5.6 a $6.0(19.2)$ & 6.1 a $6.5(22.0)$ & 6.6 a $7.0(30.3)$ & 7.1 a $7.5(14.7)$ \\
Conductividad eléctrica $\left(\mathrm{dS} \mathrm{m}^{-1}\right)$ & $<0.40(90.2)$ & 0.40 a $0.81(9.8)$ & & & \\
\hline
\end{tabular}

Cuadro 3. Niveles de contenido ( $\mathrm{mg} \mathrm{kg}^{-1}$ ) de nutrientes en el suelo y su distribución porcentual en los huertos de guayabo var. Media China en el oriente de Michoacán.

\begin{tabular}{|c|c|c|c|c|c|}
\hline \multirow{2}{*}{ Nutriente } & \multicolumn{5}{|c|}{ Nivel de contenido (porcentaje de huertos) ${ }^{+}$} \\
\hline & Bajo & Moderadamente bajo & Adecuado & Moderadamente alto & Alto \\
\hline Fósforo & $<11(26.8)$ & 11 a $20(22.7)$ & 21 a $30(13.0)$ & 31 a $40(3.4)$ & $>40(34.1)$ \\
\hline Potasio ${ }^{++}$ & $<175(17.1)$ & 175 a 250 (23.2) & 251 a 450 (46.3) & 451 a $800(8.9)$ & $>800(4.5)$ \\
\hline Calcio $^{++}$ & $<1250(5.5)$ & 1250 a $2000(11.1)$ & 2001 a $4000(53.1)$ & 4001 a 6000 (22.9) & $>6000(7.4)$ \\
\hline Magnesio $^{+\dagger}$ & $<150(0.0)$ & 150 a $250(0.0)$ & 251 a $500(11.9)$ & 501 a 1000 (48.4) & $>1000(39.7)$ \\
\hline Hierro & $<5(0.0)$ & 5 a $8(0.0)$ & 9 a $12(8.4)$ & 13 a 25 (16.5) & $>25(75.1)$ \\
\hline Manganeso & $<4(0.0)$ & 4 a $7(0.0)$ & 8 a $12(5.6)$ & 13 a $25(15.5)$ & $>25(78.9)$ \\
\hline Cobre & $<0.5(0.0)$ & 0.5 a $0.8(4.7)$ & 0.9 a $1.2(2.7)$ & 1.3 a $1.8(12.6)$ & $>1.8(80.0)$ \\
\hline Zinc & $<0.7(0.0)$ & 0.7 a $1.2(0.8)$ & 1.3 a $2.5(17.4)$ & 2.6 a $5.0(36.8)$ & $>5.0(45.0)$ \\
\hline
\end{tabular}

†De acuerdo con Castellanos et al. (2000), para los mismos métodos de análisis utilizados en la presente publicación. +†Para suelos de textura fina: arcilla, arcilla limosa, arcilla arenosa, limo. 
Cuadro 4. Factores de manejo y sus niveles mínimo, máximo y medio en los huertos de guayabo var. Media China en el oriente de Michoacán.

\begin{tabular}{|c|c|c|c|}
\hline \multirow{2}{*}{ Factor } & \multicolumn{3}{|c|}{ Nivel } \\
\hline & Mínimo & Máximo & Medio \\
\hline Edad (años) & 4 & 26 & 13 \\
\hline Número de árboles por hectárea & 204 & 625 & 360 \\
\hline Fecha de poda (día/mes) & 15/01 & $14 / 06$ & $21 / 03$ \\
\hline Días a floración desde la poda & 50 & 103 & 65 \\
\hline \multicolumn{4}{|l|}{ Riego $^{+}$} \\
\hline Número & 3 & 20 & 11 \\
\hline Intervalo (días) & 7 & 30 & 13 \\
\hline \multicolumn{4}{|l|}{ Control de malezas (número) } \\
\hline Químico & 0 & 3 & 0.75 \\
\hline Mecánico & 0 & 6 & 2.50 \\
\hline Químico-mecánico & 1 & 6 & 3.25 \\
\hline \multicolumn{4}{|l|}{ Control de plagas-enfermedades } \\
\hline Número aplicaciones de productos & 0 & 16 & 10 \\
\hline Intervalo (días) & 15 & 20 & - \\
\hline Número de fertilizaciones & 1 & 2 & 1.5 \\
\hline \multicolumn{4}{|l|}{ Cantidad de nutriente (g/árbol) } \\
\hline Nitrógeno (N): Primera $^{+\dagger}$ & 0 & 540 & 209 \\
\hline Segunda & 0 & 260 & 34 \\
\hline Fósforo $\left(\mathrm{P}_{2} \mathrm{O}_{5}\right)$ : Primera & 0 & 640 & 174 \\
\hline Segunda & 0 & 155 & 9 \\
\hline Potasio $\left(\mathrm{K}_{2} \mathrm{O}\right)$ : Primera & 0 & 540 & 166 \\
\hline Segunda & 0 & 360 & 51 \\
\hline Calcio (Ca): Primera & 0 & 896 & 178 \\
\hline Segunda & 0 & 140 & 15 \\
\hline Azufre (S): Primera & 0 & 480 & 130 \\
\hline Segunda & 0 & 56 & 3 \\
\hline Microelementos: Primera & 0 & 75 & 28 \\
\hline
\end{tabular}

†Lámina de riego variable según el tiempo de riego, de hasta $5 \mathrm{~cm}$; ${ }^{\text {†t} F e r t i l i z a c i o ́ n ~ e n ~ l a ~ p r i m e r a ~ y ~ s e g u n d a ~ a p l i c a c i o ́ n ; ~ ‘ F e r t i l i z a c i o ́ n ~ c o n ~ F e r m i l ~}$ granulado (Mg: 8.0 \%, Mn: 1.2 \%, Zn: 3.1 \%, Cu: $0.10 \%$, Fe: 2.1 \%, Ca: 3.8 \%, B: 0.40 \%, S: 9.2 \%).

para los demás factores incluidos en los modelos, con lo cual se obtuvo el rendimiento máximo o uno muy cercano a éste cuando el rango de valores de la concentración nutrimental era muy reducido en su valor superior (Cuadro 7), y partir de la disminución del rendimiento que causan las concentraciones nutrimentales bajas o altas, en el Cuadro 8 se presenta el rango de la concentración nutrimental: deficiente, bajo, adecuado, alto y excesivo para los nutrientes.

En estos términos, el rango de suficiencia nutrimental foliar presentó los siguientes valores: $\mathrm{N}, 15.0$ a $25.0 \mathrm{~g} \mathrm{~kg}^{-1}$; P, 1.7 a $3.4 \mathrm{~g} \mathrm{~kg}^{-1} ; \mathrm{K}, 10.0$ a $19.0 \mathrm{~g} \mathrm{~kg}^{-1} ; \mathrm{Ca}, 8.5$ a $14.5 \mathrm{~g} \mathrm{~kg}^{-1}$; $\mathrm{Mg}, 2.5$ a $5.0 \mathrm{~g} \mathrm{~kg}^{-1} ; \mathrm{Fe}, 100$ a $235 \mathrm{mg} \mathrm{kg}^{-1} ; \mathrm{Mn}, 70$ a $210 \mathrm{mg}$ $\mathrm{kg}^{-1} ; \mathrm{Cu}, 25$ a $65 \mathrm{mg} \mathrm{kg}^{-1} ; \mathrm{Zn}, 30$ a $52 \mathrm{mg} \mathrm{kg}^{-1} ; \mathrm{y}, \mathrm{B}, 36$ a 68 $\mathrm{mg} \mathrm{kg}^{-1}$.

El rango de concentración de nutrientes en la hoja del guayabo encontrado en la región oriente de Michoacán presentó valores mayores para $\mathrm{P}$, Fe y $\mathrm{Cu}$, menores para $\mathrm{K}$, menores y mayores para Ca y $\mathrm{Mn}$, similares y menores para Mg, similares y mayores para Zn y B, y mayores, menores y similares para $\mathrm{N}$, que los encontrados en otras regiones (Anjaneyulu y Ragahupathi, 2009; Hundal et al., 2007; Kotur et al., 1997; Natale et al., 2002; Singh y Singh, 2007), lo cual puede atribuirse a diferencias en las condiciones de suelo, clima y manejo del cultivo. 
Cuadro 5. Concentración nutrimental en la hoja, de los huertos de guayabo var. Media China a 25-50 \% de floración en el oriente de Michoacán, y su valor mínimo, máximo y medio.

\begin{tabular}{lccc}
\hline \multirow{2}{*}{ Nutriente } & \multicolumn{2}{c}{ Valor } \\
\cline { 2 - 4 } & Mínimo & Máximo & Medio \\
\hline Nitrógeno $\left(\mathrm{g} \mathrm{kg}^{-1}\right)$ & 3.5 & 27.5 & 18.0 \\
Fósforo $\left(\mathrm{g} \mathrm{kg}^{-1}\right)$ & 1.1 & 3.6 & 2.0 \\
Potasio $\left(\mathrm{g} \mathrm{kg}^{-1}\right)$ & 3.1 & 17.7 & 10.0 \\
Calcio $\left(\mathrm{g} \mathrm{kg}^{-1}\right)$ & 5.4 & 17.2 & 9.7 \\
Magnesio $\left(\mathrm{g} \mathrm{kg}^{-1}\right)$ & 1.9 & 6.2 & 3.6 \\
Hierro $\left(\mathrm{mg} \mathrm{kg}^{-1}\right)$ & 13 & 482 & 142 \\
Manganeso $\left(\mathrm{mg} \mathrm{kg}^{-1}\right)$ & 10 & 229 & 61 \\
Cobre $\left(\mathrm{mg} \mathrm{kg}^{-1}\right)$ & 3 & 192 & 43 \\
Zinc $\left(\mathrm{mg} \mathrm{kg}^{-1}\right)$ & 4 & 64 & 27 \\
Boro $\left(\mathrm{mg} \mathrm{kg}^{-1}\right)$ & 20 & 96 & 44 \\
\hline
\end{tabular}

${ }^{\dagger}$ Valores referidos a peso seco.

Cuadro 6. Modelos de regresión del rendimiento de fruto en función de la concentración nutrimental en la hoja de guayabo var. Media China a 25-50 \% de floración, para distintos nutrientes, en el oriente de Michoacán.

\begin{tabular}{lll}
\hline Nutriente & \multicolumn{1}{c}{ Modelo $^{+}$} & $\mathrm{R}^{2}$ \\
\hline Nitrógeno & $\mathrm{Y}=\ldots \ldots \ldots .+347.487 \mathrm{Fn}^{0.25}-9.345 \mathrm{Fn}$ & 0.862 \\
Fósforo & $\mathrm{Y}=\ldots \ldots \ldots .+176.642 \mathrm{Fp}^{0.5}-55.928 \mathrm{Fp}$ & 0.808 \\
Potasio & $\mathrm{Y}=\ldots \ldots \ldots .+12.602 \mathrm{Fk}-2.288 \mathrm{Fk} .5$ & 0.775 \\
Calcio & $\mathrm{Y}=\ldots \ldots \ldots .+125.140 \mathrm{Fca}^{0.5}-18.900 \mathrm{Fca}-7.061 \mathrm{~A}_{2}$ & 0.824 \\
Magnesio & $\mathrm{Y}=\ldots \ldots \ldots .+136.077 \mathrm{Fmg}^{0.5}-35.323 \mathrm{Fmg}$ & 0.885 \\
Hierro & $\mathrm{Y}=\ldots \ldots \ldots .+13.845 \mathrm{Ffe}^{0.5}-0.547 \mathrm{Ffe}$ & 0.796 \\
Manganeso & $\mathrm{Y}=\ldots \ldots \ldots .+42.732 \mathrm{Fmn}^{0.25}-0.285 \mathrm{Fmn}$ & 0.944 \\
Cobre & $\mathrm{Y}=\ldots \ldots \ldots .+364.515 \mathrm{Fcu}^{0.1}+54.239 \mathrm{Fcu}$ \\
Zinc & $\mathrm{Y}=\ldots \ldots \ldots .+2.612 \mathrm{Fzn}^{0.5}-0.0318 \mathrm{Fzn}{ }^{2}$ & 0.894 \\
Boro & $\mathrm{Y}=\ldots \ldots \ldots .+68.513 \mathrm{Fb}^{0.5}-4.811 \mathrm{Fb}$ & 0.915 \\
\hline
\end{tabular}

${ }^{\top}$ Y: rendimiento de fruto (kg/árbol); Fn, Fp, Fk, Fca y Fmg; concentraciones de N, P, K, Ca y Mg ( $\left.\mathrm{g} \mathrm{kg}^{-1}\right)$; Ffe, Fmn, Fcu, Fzn y Fb: concentraciones de Fe, Mn, Cu, Zn y B en la hoja ( $\mathrm{mg} \mathrm{kg}^{-1}$ ); $\mathrm{A}_{2}$ : variable auxiliar para el año 2008 y $\mathrm{A}_{3}$ : variable auxiliar para el año 2011, en relación al año 2006. En los modelos se excluyeron los términos correspondientes a la ordenada al origen y a las variables edad de los árboles, profundidad del suelo, número de aplicaciones para el control de enfermedades-plagas y número de veces que se practicó control de malezas, con efecto significativo en todos ellos: $\mathrm{R}^{2}$ : Coeficiente de determinación del modelo.

Según los rangos de las concentraciones nutrimentales en la hoja, en el Cuadro 9 se presenta la distribución porcentual de los huertos por rango de concentración nutrimental en la hoja. Sólo para Mg se observa un porcentaje alto de huertos $(85.9 \%)$ en el rango de concentración adecuado, y para N, P, K, Ca, Fe y B este porcentaje va de 46.0 a $68.8 \%$, con concentraciones bajas y deficientes entre 27.4 y $39.1 \%$ y de $54.0 \%$ para K; a la vez, para Mn, Cu y Zn, sólo de 23.0 a $29.5 \%$ de los huertos se encuentran en el rango de concentración adecuado, y presentan concentraciones bajas y deficientes entre 66.9 y $69.6 \%$ para $\mathrm{Zn}$ y $\mathrm{Mn}$, respectivamente, y de $53.0 \%$ para Cu. Por su parte, Cu presentó concentraciones excesivas en $18.6 \%$ de los huertos, lo que puede estar asociado con aplicaciones de productos a base de Cu (e.g. Bordocop, Cupravit Mix, Cuper Hidro) que hacen los productores antes de la floración para controlar enfermedades fungosas.

\section{Concentración nutrimental en la hoja como función de nutrientes del suelo y aplicados y factores de suelo y de manejo}

Los modelos de regresión de la concentración nutrimental en la hoja como función de, en su caso, el nutriente aplicado como fertilizante en la primera aplicación (después de la poda y antes del primer riego), contenido nutrimental y propiedades físicas y químicas del suelo y factores de manejo del cultivo, se presentan en el Cuadro 10. 
Cuadro 7. Rendimiento de fruto $(\mathrm{Y}, \mathrm{kg} / a ́ r b o l)$ en función de la concentración nutrimental en la hoja de guayabo var. Media China a $\mathbf{2 5 - 5 0} \%$ de floración en el oriente de Michoacán.

\begin{tabular}{|c|c|c|c|c|c|c|c|c|c|c|}
\hline Nutriente & & & res de $c$ & ncentra & ones nu & mentale & y rend & iento $d$ & fruto & \\
\hline Nitrógeno $\left(\mathrm{g} \mathrm{kg}^{-1}\right)$ & 7.5 & 10.0 & 12.5 & 15.0 & 17.5 & 20.0 & 22.5 & 25.0 & 27.5 & \\
\hline Y & 37.2 & 56.7 & 68.8 & 75.9 & 79.4 & 80.2 & 78.8 & 75.6 & 71.0 & \\
\hline Fósforo $\left(\mathrm{g} \mathrm{kg}^{-1}\right)$ & 1.0 & 1.3 & 1.6 & 1.9 & 2.2 & 2.5 & 2.8 & 3.1 & 3.4 & \\
\hline Y & 61.3 & 69.3 & 74.6 & 77.9 & 79.6 & 80.1 & 79.6 & 78.3 & 76.2 & \\
\hline Potasio $\left(\mathrm{g} \mathrm{kg}^{-1}\right)$ & 5.0 & 7.0 & 9.0 & 11.0 & 13.0 & 15.0 & 17.0 & 19.0 & 21.0 & \\
\hline$Y$ & 58.4 & 67.4 & 74.0 & 78.3 & 80.6 & 81.0 & 80.6 & 77.0 & 72.6 & \\
\hline Calcio $\left(\mathrm{g} \mathrm{kg}^{-1}\right)$ & 5.0 & 7.0 & 9.0 & 1.0 & 11.0 & 12.0 & 13.0 & 14.0 & 15.0 & 16.0 \\
\hline$Y$ & 56.1 & 70.5 & 77.8 & 79.6 & 80.3 & 80.2 & 79.4 & 77.8 & 75.7 & 73.0 \\
\hline Magnesio $\left(\mathrm{g} \mathrm{kg}^{-1}\right)$ & 2.0 & 2.5 & 3.0 & 2.5 & 4.0 & 4.5 & 5.0 & 5.5 & 6.0 & \\
\hline Y & 71.2 & 76.3 & 79.1 & 80.4 & 80.3 & 79.1 & 77.1 & 74.3 & 70.8 & \\
\hline Hierro $\left(\mathrm{mg} \mathrm{kg}^{-1}\right)$ & 40.0 & 70.0 & 100.0 & 130.0 & 160.0 & 190.0 & 220.0 & 250.0 & 280.0 & 310.0 \\
\hline Y & 57.7 & 69.6 & 75.8 & 78.7 & 79.6 & 78.9 & 77.0 & 74.1 & 70.4 & 66.1 \\
\hline Manganeso $\left(\mathrm{mg} \mathrm{kg}^{-1}\right)$ & 20.0 & 40.0 & 60.0 & 80.0 & 100.0 & 120.0 & 140.0 & 160.0 & 180.0 & 200.0 \\
\hline Y & 57.6 & 69.0 & 74.0 & 78.0 & 79.6 & 80.2 & 80.1 & 79.4 & 78.2 & 76.7 \\
\hline Cobre $\left(\mathrm{mg} \mathrm{kg}^{-1}\right)$ & 5.0 & 15.0 & 25.0 & 35.0 & 45.0 & 55.0 & 65.0 & 75.0 & 85.0 & 95.0 \\
\hline Y & 65.9 & 71.3 & 76.7 & 79.5 & 80.2 & 79.2 & 76.8 & 73.2 & 68.6 & 63.2 \\
\hline Zinc $\left(\mathrm{mg} \mathrm{kg}^{-1}\right)$ & 6.0 & 13.0 & 20.0 & 27.0 & 34.0 & 41.0 & 48.0 & 55.0 & 62.0 & \\
\hline Y & 42.4 & 56.5 & 67.4 & 75.2 & 78.0 & 81.5 & 80.0 & 75.4 & 67.6 & \\
\hline Boro $\left(\mathrm{mg} \mathrm{kg}^{-1}\right)$ & 25.0 & 35.0 & 45.0 & 55.0 & 65.0 & 75.0 & 85.0 & 95.0 & & \\
\hline Y & 58.5 & 73.3 & 79.5 & 80.0 & 76.3 & 69.2 & 59.6 & 49.7 & & \\
\hline
\end{tabular}

${ }^{\dagger}$ Valores referidos a peso seco; Y: rendimiento (kg/árbol).

Cuadro 8. Rango de la concentración nutrimental en la hoja de guayabo var. Media China a $25-50 \%$ de floración en el oriente de Michoacán.

\begin{tabular}{|c|c|c|c|c|c|}
\hline \multirow{2}{*}{ Nutriente } & \multicolumn{5}{|c|}{ Rango de concentración nutrimental $^{+}$} \\
\hline & Deficiente & Bajo & Adecuado & Alto & Excesivo \\
\hline Nitrógeno $\left(\mathrm{g} \mathrm{kg}^{-1}\right)$ & $<13.5$ & 13.5 a 15.0 & 15.0 a 25.0 & 25.0 a 27.0 & - \\
\hline Fósforo $\left(\mathrm{g} \mathrm{kg}^{-1}\right)$ & $<1.4$ & 1.4 a 1.7 & 1.7 a 3.4 & $>3.4$ & - \\
\hline Potasio $\left(\mathrm{g} \mathrm{kg}^{-1}\right)$ & $<8.0$ & 8.0 a 10.0 & 10.0 a 19.0 & $>19.0$ & - \\
\hline Calcio $\left(\mathrm{g} \mathrm{kg}^{-1}\right)$ & $<7.5$ & 7.5 a 8.5 & 8.5 a 14.5 & 14.5 a 16.0 & $>16.0$ \\
\hline Magnesio $\left(\mathrm{g} \mathrm{kg}^{-1}\right)$ & $<2.0$ & 2.0 a 2.5 & 2.5 a 5.0 & 5.0 a 6.0 & $>6.0$ \\
\hline Hierro $\left(\mathrm{mg} \mathrm{kg}^{-1}\right)$ & $<75$ & 75 a 100 & 100 a 235 & 235 a 275 & - \\
\hline Manganeso $\left(\mathrm{mg} \mathrm{kg}^{-1}\right)$ & $<50$ & 50 a 70 & 70 a 210 & 210 a 250 & - \\
\hline Cobre $\left(\mathrm{mg} \mathrm{kg}^{-1}\right)$ & $<15$ & 15 a 25 & 25 a 65 & 65 a 80 & $>80$ \\
\hline Zinc $\left(\mathrm{mg} \mathrm{kg}^{-1}\right)$ & $<25$ & 25 a 30 & 30 a 52 & 52 a 57 & $>57$ \\
\hline Boro $\left(\mathrm{mg} \mathrm{kg}^{-1}\right)$ & $<30$ & 30 a 36 & 36 a 68 & 68 a 76 & - \\
\hline
\end{tabular}

${ }^{\top}$ Valores referidos a peso seco.

Para los nutrientes N, P, K y Ca, aplicados en la primera fertilización de los árboles, la concentración en la hoja fue incrementada por las respectivas cantidades aplicadas de fertilizantes y para $\mathrm{N}$ también por la materia orgánica del suelo, y para $\mathrm{P}, \mathrm{K}$ y Ca también por los respectivos contenidos nutrimentales del suelo. Para el Mg, aplicado en cierta cantidad como Fermil (8.0 \% de Mg) en la primera fertilización, la concentración en la hoja fue incrementada sólo por su contenido en el suelo. Para los microelementos Fe, Mn, Cu y Zn, aplicados en la primera fertilización como Fermil (2.1\% de Fe, $1.2 \%$ de Mn, $0.10 \%$ de $\mathrm{Cu}, 3.1 \%$ de Zn), sólo para el Mn y Cu se observó un efecto de este fertilizante de 
Cuadro 9. Distribución de los huertos por rango de la concentración nutrimental en la hoja de guayabo var. Media China a 25-50 \% de floración en el oriente de Michoacán.

\begin{tabular}{lccccc}
\hline \multirow{2}{*}{ Nutriente } & \multicolumn{5}{c}{ Porcentaje de huertos por rango } \\
\cline { 2 - 6 } & Deficiente & Bajo & Adecuado & Alto & Excesivo \\
\hline Nitrógeno & 20.3 & 11.5 & 60.2 & 8.0 & - \\
Fósforo & 6.2 & 23.0 & 68.1 & 2.7 & - \\
Potasio & 23.0 & 31.0 & 46.0 & - & - \\
Calcio & 21.2 & 15.9 & 54.9 & 2.7 & 5.3 \\
Magnesio & 0.9 & 8.8 & 85.9 & 3.5 & 0.9 \\
Hierro & 14.2 & 25.7 & 49.5 & 10.6 & - \\
Manganeso & 49.1 & 20.5 & 29.5 & 0.9 & - \\
Cobre & 31.9 & 22.1 & 23.0 & 4.4 & 18.6 \\
Zinc & 57.5 & 9.7 & 26.6 & 4.4 & 1.8 \\
Boro & 17.7 & 9.7 & 65.5 & 7.1 & - \\
\hline
\end{tabular}

Cuadro 10. Modelos de regresión de la concentración nutrimental en la hoja de guayabo var. Media China a 25-50 \% de floración, en función del nutriente aplicado como fertilizante, contenido nutrimental y propiedades químicas del suelo, y factores de manejo del cultivo en el oriente de Michoacán.

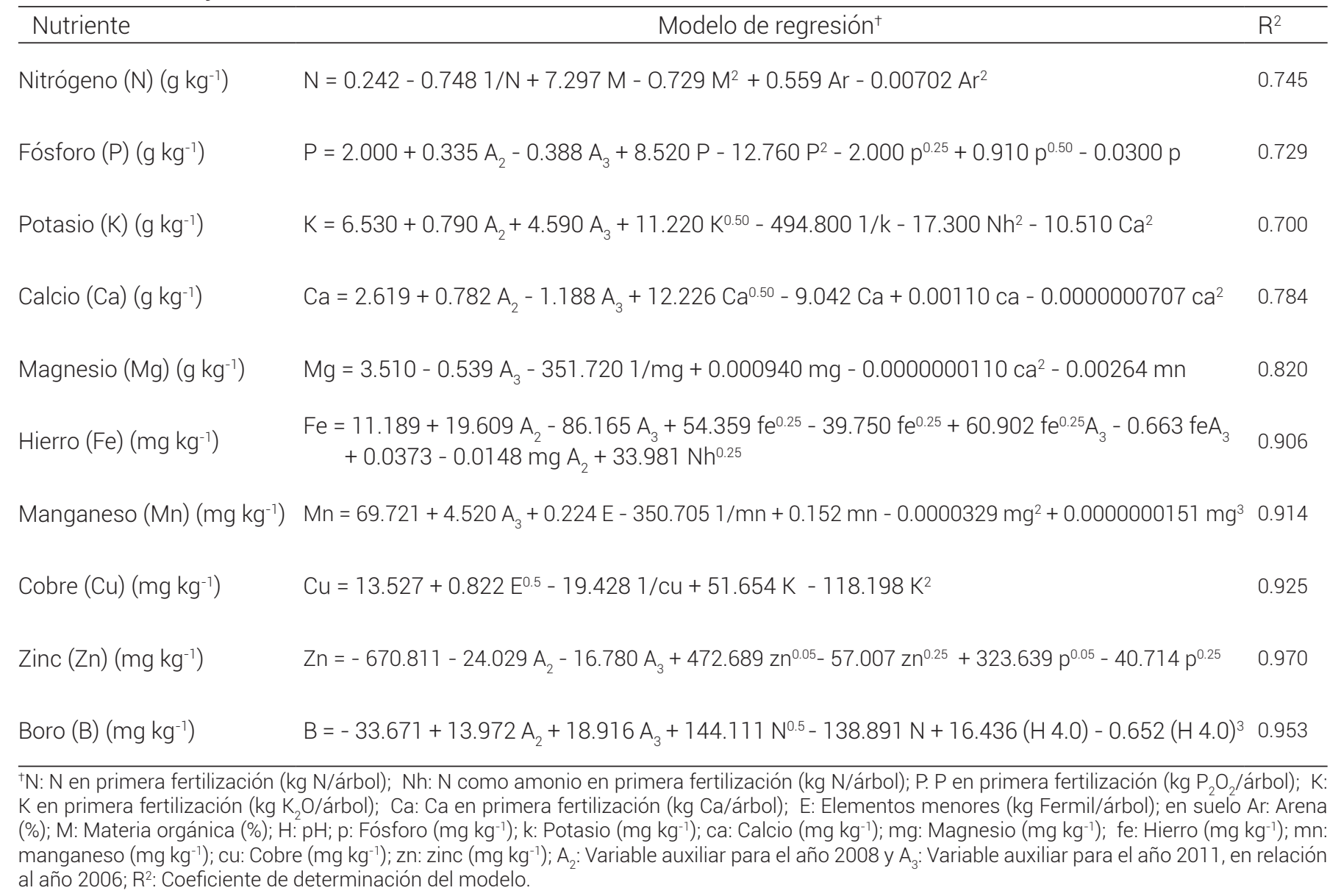


incrementar la concentración en la hoja, y para todos ellos esta concentración fue incrementada por sus respectivos contenidos en el suelo. Para el B, también presente en el Fermil, la concentración en la hoja no fue incrementada por este fertilizante, pero no se determinó su contenido en el suelo (Cuadro 10).

Por otra parte, se observó un efecto de nutrientes presentes en el suelo o aplicados como fertilizantes, sobre la absorción de otros nutrientes por la planta. Tal fue el caso de un efecto negativo de los fertilizantes amoniacales y cálcicos sobre absorción de $\mathrm{K}$, negativo del Mg del suelo sobre la absorción de Fe y Mn, positivo de los fertilizantes amoniacales sobre la absorción de Fe, positivo de los fertilizantes potásicos sobre la absorción de $\mathrm{Cu}$, negativo del $P$ del suelo a contenidos altos sobre la absorción de Zn y positivo de los fertilizantes nitrogenados sobre la absorción de B (Cuadro 10).

Este efecto normalmente se debe a fenómenos sinérgicos o antagónicos en la absorción por la planta, que ocurren ya sea por reacciones a nivel del suelo o en la superficie de la raíz (Castellanos et al., 2000; Havlin et al., 1999), en lo que puede influir la forma química del nutriente, como es el caso del N, ya sea como ión amonio o como ión nitrato.

Otros aspectos observados fueron el efecto positivo del $\mathrm{pH}$ del suelo sobre la absorción de B, hasta un valor de 6.9, y el efecto positivo de la arena del suelo, hasta un valor de 40 \%, sobre la absorción de N por la planta (Cuadro 10).

El efecto de factores distintos al de los fertilizantes y contenido nutrimental del suelo sobre la absorción de los nutrientes por las plantas, como propiedades físicas, textura en el presente caso, y químicas del suelo, así como de factores de manejo del cultivo, ha sido observado en diversas condiciones de producción (Castellanos et al., 2000; Havlin et al., 1999).

Con los modelos de regresión obtenidos (Cuadro 10) se estimaron las concentraciones nutrimentales en la hoja como función de, según fuere el caso, las cantidades de fertilizante aplicadas en la primera fertilización y contenidos nutrimentales del suelo, y del pH del suelo para el $\mathrm{B}, \mathrm{a}$ valores de los demás factores que afectaron la absorción nutrimental medios o de mayor frecuencia, y para el caso de los fertilizantes, las cantidades recomendadas en la zona o cercanas a ella (Volke-Haller et al., 2012), según se indica en el Cuadro 11.

En la Región Oriente de Michoacán se han determinado las cantidades de N, P, K, Ca y microelementos (Fermil) a aplicar en la primera fertilización del guayabo, las cuales son de $200 \mathrm{~g} \mathrm{~N} / a ́ r b o l, 150$ a 175 g P $\mathrm{O}_{5} /$ árbol, $200 \mathrm{~g} \mathrm{~K}_{2} \mathrm{O} /$ árbol, 150 g Ca/árbol y 75 g Fermil/árbol, y para suelos con contenidos de $\mathrm{P}$ menores de $10 \mathrm{mg} \mathrm{kg}^{-1}$, la dosis aumenta a $200 \mathrm{~g} \mathrm{P}_{2} \mathrm{O}_{5} /$ árbol, y con contenidos de Ca de 1250 a 2000 $\mathrm{mg} \mathrm{kg}{ }^{-1}$ y menores de $1250 \mathrm{mg} \mathrm{kg}^{-1}$, la dosis aumenta a 175 y $200 \mathrm{~g} \mathrm{Ca/árbol,} \mathrm{respectivamente} \mathrm{(Volke-Haller} \mathrm{et} \mathrm{al.,}$ 2012).

Las concentraciones de $\mathrm{N}$ en la hoja corresponden a 1.5 y $2.5 \%$ de materia orgánica del suelo como cantidades más frecuentes (Cuadro 2), y con una aplicación de $200 \mathrm{~g}$ N/árbol en la primera fertilización (Volke-Haller et al., 2012) se alcanzaron concentraciones foliares de N de 15.4 y $19.8 \mathrm{~g} \mathrm{~kg}^{-1}$, respectivamente, valores similares y mayores al límite inferior del rango de concentración adecuada de $15.0 \mathrm{~g} \mathrm{~kg}^{-1}$ (Cuadro 8), de tal modo que para suelos con menores contenidos de materia orgánica se puede aumentar la aplicación de $\mathrm{N}$ y con mayores contenidos de materia orgánica disminuir la aplicación de $\mathrm{N}$.

Las concentraciones de P en la hoja corresponden a 10 y $25 \mathrm{mg} \mathrm{P} \mathrm{kg}^{-1}$ del suelo como contenidos bajos y moderadamente bajos, y adecuados, respectivamente (Cuadro 3), y con aplicaciones de 150 a $175 \mathrm{~g} \mathrm{P}_{2} \mathrm{O}_{5} /$ árbol en la primera fertilización (Volke-Haller et al., 2012), se alcanzaron concentraciones foliares de P de 2.0 a $2.4 \mathrm{~g} \mathrm{~kg}^{-1}$, superiores al límite inferior del rango de concentración adecuada de 1.7 $\mathrm{g} \mathrm{kg}^{-1}$ (Cuadro 8), por lo que se puede disminuir la cantidad de $\mathrm{P}$ que se recomienda aplicar en la primera fertilización.

Las concentraciones de K en la hoja corresponden a 175 y $350 \mathrm{mg} \mathrm{K} \mathrm{kg}^{-1}$ del suelo como valores más frecuentes (Cuadro 3), y con aplicaciones de $200 \mathrm{~g} \mathrm{~K}_{2} \mathrm{O} / \mathrm{a} r b o l$ en la primera fertilización (Volke-Haller et al., 2012) se alcanzó una concentración de $\mathrm{K}$ en la hoja de $10.0 \mathrm{~g} \mathrm{~kg}^{-1}$, valor mínimo de la concentración adecuada (Cuadro 8); por lo tanto, para suelos con contenidos menores de $\mathrm{K}$ esta cantidad sería adecuada, y podría disminuirse en suelos con mayores contenidos de $\mathrm{K}$.

La concentración adecuada de Ca en la hoja, de 8.5 a más de $14.5 \mathrm{~g} \mathrm{~kg}^{-1}$ (Cuadro 8), se alcanzó con aplicaciones de 150 a $175 \mathrm{~g} \mathrm{Ca/árbol} \mathrm{en} \mathrm{la} \mathrm{primera} \mathrm{fertilización} \mathrm{(Volke-}$ Haller et al., 2012) para un contenido medio de Ca del suelo de $3000 \mathrm{mg} \mathrm{kg}^{-1}$ como valor más frecuente (Cuadro 3);

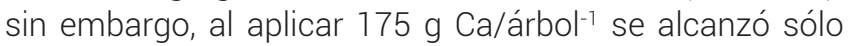
una concentración foliar de Ca de $8.6 \mathrm{~g} \mathrm{~kg}^{-1}$, por lo que en suelos con contenidos de calcio menores de $2000 \mathrm{mg} \mathrm{kg}^{-1}$, habría que considerar fertilizaciones mayores de $150 \mathrm{~g} \mathrm{Ca/}$ árbol.

Para Mg, 90.3 \% de los huertos presentaron una concentración adecuada o más alta en la hoja (Cuadro 9), lo que coincide con contenidos adecuados a altos de este nutriente en el suelo (Cuadro 3); además, este elemento 
Cuadro 11. Cantidad de nutriente a aplicar o contenido de nutriente en el suelo, necesario para alcanzar la concentración nutrimental adecuada en la hoja de guayabo var. Media China a 25-50 \% de floración en el oriente de Michoacán.

\begin{tabular}{|c|c|c|c|c|c|c|c|c|}
\hline \multirow{2}{*}{$\begin{array}{l}\text { Nutriente } \\
\text { Nitrógeno }\end{array}$} & \multicolumn{8}{|c|}{$\begin{array}{c}\text { Concentración nutrimental en hoja }{ }^{+} \text {y nutriente aplicado o contenido } \\
\text { del nutriente en el suelo }\end{array}$} \\
\hline & & & & & & & & \\
\hline Foliar ( $\left.\mathrm{g} \mathrm{kg}^{-1}\right)$ : (suelo, 1.5\% MO^) & 4.2 & 11.7 & 14.1 & 14.9 & 15.4 & 15.8 & 16.1 & 16.4 \\
\hline (suelo, $2.5 \% \mathrm{MO}$ ) & 8.6 & 16.0 & 18.5 & 19.2 & 19.8 & 20.2 & 20.5 & 20.8 \\
\hline Fertilizante (g N/árbol) & 50.0 & 100.0 & 150.0 & 175.0 & 200.0 & 225.0 & 250.0 & 275.0 \\
\hline \multicolumn{9}{|l|}{ Fósforo } \\
\hline Foliar ( $\mathrm{g} \mathrm{kg}^{-1}$ ): (suelo, 10 mg P kg-1) & 10.0 & 14.0 & 17.0 & 18.0 & 20.0 & 21.0 & 22.0 & 22.0 \\
\hline (suelo, $25 \mathrm{mg} \mathrm{P} \mathrm{kg}^{-1}$ ) & 13.0 & 17.0 & 20.0 & 21.0 & 23.0 & 24.0 & 25.0 & 26.0 \\
\hline Fertilizante ( $\mathrm{g} \mathrm{P}_{2} \mathrm{O}_{5} /$ árbol) & 0.0 & 50.0 & 100.0 & 125.0 & 150.0 & 175.0 & 200.0 & 225.0 \\
\hline \multicolumn{9}{|l|}{ Potasio } \\
\hline Foliar (g kg-1): (suelo, $175 \mathrm{mg} \mathrm{K} \mathrm{kg}^{-1}$ ) & 7.6 & 8.2 & 8.7 & 9.1 & 9.5 & 9.8 & 10.2 & 10.8 \\
\hline (suelo, 350 mg K kg-1) & 9.1 & 9.7 & 10.2 & 10.6 & 11.0 & 11.3 & 11.7 & 12.2 \\
\hline Fertilizante ( $\left.\mathrm{g} \mathrm{K}_{2} \mathrm{O} / a ́ r b o l\right)$ & 50.0 & 75.0 & 100.0 & 125.0 & 150.0 & 175.0 & 200.0 & 250.0 \\
\hline \multicolumn{9}{|l|}{ Calcio } \\
\hline Foliar $\left(\mathrm{g} \mathrm{kg}^{-1}\right)$ & 7.4 & 7.8 & 8.1 & 8.3 & 8.5 & 8.6 & 8.8 & 8.9 \\
\hline Fertilizante (g Ca/árbol) & 50.0 & 75.0 & 100.0 & 125.0 & 150.0 & 175.0 & 200.0 & 225.0 \\
\hline \multicolumn{9}{|l|}{ Magnesio } \\
\hline Foliar $\left(\mathrm{g} \mathrm{kg}^{-1}\right)$ & 1.9 & 2.9 & 3.3 & 3.7 & 4.0 & 4.3 & 4.6 & 4.8 \\
\hline En suelo $\left(\mathrm{mg} \mathrm{kg}^{-1}\right)$ & 250.0 & 500.0 & 750.0 & 1000.0 & 1250.0 & 1500.0 & 1750.0 & 2000.0 \\
\hline \multicolumn{9}{|l|}{ Hierro } \\
\hline Foliar $\left(\mathrm{mg} \mathrm{kg}^{-1}\right)$ & 77.0 & 94.0 & 112.0 & 124.0 & 132.0 & 139.0 & 150.0 & 157.0 \\
\hline En suelo $\left(\mathrm{mg} \mathrm{kg}^{-1}\right)$ & 5.0 & 10.0 & 20.0 & 30.0 & 40.0 & 50.0 & 75.0 & 100.0 \\
\hline \multicolumn{9}{|l|}{ Manganeso } \\
\hline Foliar $\left(\mathrm{mg} \mathrm{kg}^{-1}\right)^{9}$ & - & 43.0 & 62.0 & 69.0 & 74.0 & 77.0 & 80.0 & 82.0 \\
\hline En suelo (mg kg-1) & 5.0 & 10.0 & 20.0 & 30.0 & 40.0 & 50.0 & 60.0 & 70.0 \\
\hline \multicolumn{9}{|l|}{ Cobre } \\
\hline Foliar $\left(\mathrm{mg} \mathrm{kg}^{-1}\right)^{\hookrightarrow}$ & - & 17.0 & 26.0 & 30.0 & 31.0 & 32.0 & 33.0 & 33.0 \\
\hline En suelo (mg kg-1) & 0.5 & 1.0 & 2.0 & 3.0 & 4.0 & 5.0 & 6.0 & 7.0 \\
\hline \multicolumn{9}{|l|}{ Zinc } \\
\hline Foliar $\left(\mathrm{mg} \mathrm{kg}^{-1}\right)^{+}$ & 12.0 & 19.0 & 25.0 & 28.0 & 30.0 & 31.0 & 32.0 & 32.0 \\
\hline En suelo $\left(\mathrm{mg} \mathrm{kg}^{-1}\right)$ & 0.5 & 1.0 & 2.0 & 3.0 & 4.0 & 5.0 & 6.0 & 7.0 \\
\hline \multicolumn{9}{|l|}{ Borox } \\
\hline Foliar (mg kg-1) & 29.0 & 36.0 & 41.0 & 44.0 & 45.0 & 43.0 & & \\
\hline pH del suelo & 5.0 & 5.5 & 6.0 & 6.5 & 7.0 & 7.5 & & \\
\hline
\end{tabular}

†Valores referidos a peso seco. ${ }^{\dagger}$ Los valores considerados de los factores incluidos en los modelos de regresión del Cuadro 10, ajenos al correspondiente fertilizante o nutriente en el suelo para el cual se calculó la concentración nutrimental en hoja, fueron los siguientes: $\mathrm{N}$ (primera fertilización) $=175 \mathrm{~g} \mathrm{~N} / a ́ r b o l, ~ N h$ (primera fertilización) $=175 \mathrm{~g} \mathrm{~N} / a ́ r b o l, ~ P$ (primera fertilización) = $175 \mathrm{~g} \mathrm{P} \mathrm{O}_{5} /$ árbol, $\mathrm{K}$ (primera fertilización) = $200 \mathrm{~g}$ $\mathrm{K}_{2} \mathrm{O} /$ árbol, $\mathrm{Ca}$ (primera fertilización) $=175 \mathrm{~g} \mathrm{Ca} /$ árbol, elementos menores (primera fertilización) $=75 \mathrm{~g}$ Fermil/árbol, suelo: arena $=25 \%$, materia orgánica $=2.5 \%$, fósforo $=25 \mathrm{mg} \mathrm{kg}^{-1}$, potasio $=\mathrm{mg} \mathrm{kg}^{-1}$, calcio $=3000 \mathrm{mg} \mathrm{kg}^{-1}$, magnesio $=750 \mathrm{mg} \mathrm{kg}^{-1}$, manganeso $=40 \mathrm{mg} \mathrm{kg}^{-1}$. ${ }^{\top} \mathrm{MO}$ : $\mathrm{materia}^{2}$ orgánica de suelo. "Con $75 \mathrm{~g}$ Fermil/árbol. № se determinó el contenido de B en el suelo. 
se encuentra en el Fermil (8 \% de Mg) que se aplica en la primera fertilización a razón de 75 g Fermil/árbol.

En el caso de Fe, Mn, Cu y Zn, la concentración adecuada en la hoja se alcanzó con contenidos en el suelo de 15, 35, 2.0 y $2.0 \mathrm{mg} \mathrm{kg}^{-1}$, respectivamente, más aplicaciones de 75 $\mathrm{g}$ Fermil/árbol en la primera fertilización para el Mn y Cu, y dichos contenidos se encuentran en 81.6, 94.0, 80.0 y 81.8 $\%$ de los huertos, respectivamente (Cuadro 3 ); sin embargo, 39.9, 69.6, 54.0 y $67.0 \%$ de los huertos presentaron concentraciones en la hoja bajas o deficientes (Cuadro 9), por lo que se deduce que no existe una adecuada relación entre los valores nutrimentales en hoja y los contenidos de estos nutrientes en el suelo.

La concentración adecuada de B en la hoja fue mayor de $36 \mathrm{mg} \mathrm{kg}^{-1}$, alcanzada con pH del suelo entre 5.6 y 7.5 , y en $92.6 \%$ de los huertos (Cuadro 9), con un valor máximo para el pH de 6.9; no obstante, $27.4 \%$ de los huertos mostraron concentraciones foliares bajas o deficientes de $\mathrm{B}$, por lo que además del pH puede existir algún otro factor que afecta la absorción del B por la planta, el cual no se detectó en el presente estudio.

\section{CONCLUSIONES}

Las concentraciones nutrimentales adecuadas determinadas en la hoja del guayabo fueron para: $\mathrm{N}, 15.0$ a $25.0 \mathrm{~g}$ $\mathrm{kg}^{-1} ; \mathrm{P}, 1.7$ a $3.4 \mathrm{~g} \mathrm{~kg}^{-1} ; \mathrm{K}, 10.0$ a $19.0 \mathrm{~g} \mathrm{~kg}^{-1}$; Ca, 8.5 a 14.5 $\mathrm{g} \mathrm{kg}^{-1} ; \mathrm{Mg}, 2.5$ a $5.0 \mathrm{~g} \mathrm{~kg}^{-1} ; \mathrm{Fe}, 100$ a $235 \mathrm{mg} \mathrm{kg}^{-1} ; \mathrm{Mn}, 70$ a $210 \mathrm{mg} \mathrm{kg}^{-1}$; $\mathrm{Cu}, 25$ a $65 \mathrm{mg} \mathrm{kg}^{-1}$; Zn, 30 a $52 \mathrm{mg} \mathrm{kg}^{-1}$; y B, 36 a $68 \mathrm{mg} \mathrm{kg}^{-1}$. Las concentraciones nutrimentales de $\mathrm{N}, \mathrm{P}, \mathrm{K}, \mathrm{Ca}, \mathrm{Mn}$ y $\mathrm{Cu}$ en la hoja, estuvieron asociadas con la respectiva fertilización, los contenidos de suelo para todos los nutrientes excepto $B$, y para todos los nutrientes con algunos factores de suelo y de manejo del cultivo que favorecieron o desfavorecieron la absorción nutrimental por la planta. El rango de suficiencia nutrimental adecuado obtenido para los nutrientes considerados se asoció con contenidos nutrimentales moderadamente altos y altos del suelo, y las cantidades de fertilizantes recomendadas a aplicar en la región. El rango de suficiencia nutrimental adecuado determinado para los nutrientes considerados presentó diferencias al observado en otras regiones productoras de guayaba.

\section{BIBLIOGRAFÍA}

Alcántar G. G. y M. Sandoval V. (1999) Manual de Análisis Químico de Tejido Vegetal. Guía de Muestreo, Preparación, Análisis e Interpretación. Publicación Especial Núm. 10. Sociedad Mexicana de la Ciencia del Suelo, A.C. Chapingo, Edo. de México, México. $156 \mathrm{p}$.

Anjaneyulu K. and H. B. Raghupathi (2009) Identification of yield-limiting nutrients through DRIS leaf nutrient norms indices in guava (Psidium guajava). Indian Journal of Agricultural Sciences
79:418-421

Bates T. E. (1971) Factors affecting critical nutrient concentrations in plants and their evaluation: a review. Soil Science 112:116-130.

Castelán-Estrada M. y A. E. Becerril-Román (2004) Fisiología de la producción forzada en guayaba. II. Nutrientes y respuesta floral. Interciencia 29:680-685

Castellanos J. Z., J. X. Uvalle-Bueno y A. Aguilar-Santelises (2000) Manual de Interpretación de Análisis de Suelos y Aguas. 2a ed. Instituto de Capacitación para la Productividad Agrícola. San Miguel de Allende, Guanajuato, México. 226 p.

Damián-Nava A., V. A. González-Hernández, P. Sánchez-García, C. Peña-Valdivia y M. Livera-Muñoz (2006) Dinámica y diagnóstico nutrimental del guayabo en Iguala, Guerrero, México. Terra Latinoamericana 24:125-132

Etchevers B. J. D. y J. Padilla (2012) Nutrición de cultivos. In: Diagnóstico de la Fertilidad del Suelo. G. Alcántar G. y L. I. Trejo-Téllez. (coords.) Colegio de Postgraguados. Montecillo, Edo. de México, México. pp:249-272.

Havlin J. L., J. D. Beaton, S. L. Tisdale and W. L. Nelson (1999) Soil Fertility and Fertilizers. An Introduction to Nutrient Management. 6th ed. Prentice Hall. New York, USA. $487 \mathrm{p}$

Hundal H. S., D. Singh and K. Singh (2007) Monitoring nutrient status of guava fruit trees in Punjab, Northwest India, through the diagnostic and recommendation integrated system approach. Communications in Soil Science and Plant Analysis 38:21172130.

INEGI, Instituto Nacional de Estadística, Geografía e Informática (2009) Prontuario de información geográfica municipal de los Estados Unidos Mexicanos. Juárez/Jungapeo/Zitácuaro, Michoacán de Ocampo. Instituto Nacional de Estadística, Geografía e Informática. Aguascalientes, Aguascalientes, México. http://www3. inegi.org.mx/contenidos/app/mexicocifras/datos_geograficos/16/16046.pdf (Enero 2017).

Jones J. B. (1985) Soil testing and plant analysis: guides to the fertilization of horticultural crops. Horticultural Reviews 7:1-68.

Kotur S. C., Ramkumar and H. P. Singh (1997) Influence of nitrogen, phosphorus and potassium on composition of leaf and its relationship with fruit yield in 'Allahabad Safeda' guava (Psidium guajava L.) on an Alfisol. Indian Journal of Agricultural Sciences 67:568-570.

Malavolta E., G. C. Vitli e S. A. de Oliveira (1997) Avaliação do Estado Nutricional das Plantas: Principios e Aplicações. 2a ed. Associação Brasileira para Pesquisa da Potassa e do Fosfato. Piracicaba, Brasil. $319 \mathrm{p}$.

Marchal J. (1984) Guava. In: Plant Analysis as a Guide to the Nutrient Requirements of Temperate and Tropical Crops. P. MartinPrével, J. Gagnard and P. Gautier (eds.). Translated from the French by M. R. J. Holmes. Lavoisier Publishing Inc. Paris, France. pp:440-443.

Medina G. G., J. A. Ruíz C. y R. A. Martínez P. (1998) Los Climas de México: una Estratificación Ambiental Basada en el Componente Climático. Libro Técnico Núm. 1. Instituto Nacional de Investigaciones Forestales, Agrícolas y Pecuarias. Uruapan, Michoacán, México. 103 p.

Mendoza L. M. R., A. Luis A., S. F. Castillo 0. e I. Vidales F. (2005) Diagnóstico del Manejo Actual del Cultivo de Guayaba en la Región Oriente de Michoacán. Publicación Especial Núm. 1. Instituto Nacional de Investigaciones Forestales, Agrícolas y Pecuarias. Uruapan, Michoacán, México. 49 p.

Mills H. A. and J. B. Jones (1996) Plant Analysis Hanbook II: A Practical Samplig, Preparation, Analysis and Interpretation Guide. MicroMacro Publishing, Inc. Athens, GA, USA. 422 p.

Natale W., E. L. M. Coutinho, F. M. Pereira and A. E. Boaretto (2002) Nutrient foliar content for high productivity cultivars of guava in Brazil. Acta Horticulturae 594:383-386.

Otero S. M. A., A. E. Becerril R., G. Alcántar G. y R. Mosqueda V. (1997) Producción forzada de guayabo en invernadero. Agrociencia 31:285-290.

Reuter D. J., J. B. Robinson, K. I. Peverill, G. H. Price and M. J. Lambert (1997) Guidelines for collecting, handling and analyzing plant materials. In: Plant Analysis. An Interpretation Manual. 2nd ed. D. J. Reuter and J. B. Robinson (eds.). CSIRO Publishing. Collingwood, Australia. pp:55-70 
Sánchez R. G. (2007) La Red de Valor Guayaba en el Oriente del Estado de Michoacán: Bases para su Desarrollo Regional Competitivo y Sustentable. Fundación Produce Michoacán. Morelia, Michoacán, México. 134 p.

Sánchez G. P., C. Molinos S., G. Alcántar G. y M. Sandoval V. (2012) Diagnóstico nutrimental en plantas. In: Nutrición de Cultivos. G. Alcántar G. y L. I. Trejo-Téllez (coords.). Colegio de Postgraduados. Montecillo, Edo. de México, México. pp:201-247.

SEMARNAT, Secretaría del Medio Ambiente y Recursos Naturales (2002) Norma Oficial Mexicana NOM-021-RECNAT 2000, que establece las especificaciones de fertilidad, salinidad y clasificación de suelos. Estudio, muestreo y análisis. Diario Oficial (2a ed.), 31 de diciembre de 2002. México D.F., México.

SIAP, Servicio de Información y Estadística Agroalimentaria y Pesquera (2014) Producción Agrícola, Guayaba. Secretaría de Agricultura, Ganadería Desarrollo Rural, Pesca y Alimentación. México. http:// infosiap.siap.gob.mx:8080/agricola_siap_gobmx/ResumenDelegacion.do (Enero, 2016).
Singh H. P. and G. Singh (2007) Nutrient and water management in guava. Acta Horticulturae 735:389-397.

SMN, Servicio Meteorológico Nacional (2014) Información Climatológica. Normales Climatológicas. Servicio Meteorológico Nacional. Michoacán, México.http://smn.cna.gob.mx/es/component/content/ article?id=42 (Enero 2016)

Trajano Maia J. L., L. H. Bassoi, D. J. Silva, M. A. Coelho de Lima, J. S. de Assis and P. L. Dantas de Morais (2007) Assessment on nutrient levels in the aerial biomass of irrigated guava in São Francisco Valley, Brazil. Revista Brasileira de Fruticultura 29:705-709.

Volke-Haller V. (2008) Estimación de Funciones de Respuesta para Información de Tipo no Experimental, Mediante Regresión. Colegio de Postgraduados. Montecillo, Edo. de México, México. 113 p.

Volke-Haller V. H., R. Camacho-Blas, P. Sánchez-García, A. Rebollar-Alviter y F. Ruíz-Ramírez (2012) Manual de Producción de Guayaba en la Región Oriente del Estado de Michoacán. Sistema Producto Guayaba - Coordinadora de las Fundaciones Produce, A. C. Colegio de Postgraduados. Montecillo, Edo. de México, México. $107 \mathrm{p}$ 
